\title{
Infezioni del Sistema Nervoso centrale da Coxsackievirus ed Echovirus - Risultati di 5 anni di osservazione nel territorio Piemontese
}

Pietro Giorgio Pistono, Morena Martorana, Lidia Allegramente, Maria Grazia Milia, Antonio Di Garbo, Vincenzo Bossi, Maria Teresa Granito, Paola Russo, Francesca Piro

Ospedale Amedeo di Savoia (ASL 3) - Torino

Key words: Non Polio Enterovirus, AAM, encephalitits,

\section{SUMMARY}

The report is an overview of enterovirus epidemiology in Piemonte during a 5-year period from 2000 to 2004 with an investigative protocol recording epidemiologic, clinical, and laboratory data. A total of 1232 clinical cerebrospinal fluid (CSF) were collected from patients having clinical manifestations of aseptic meningitis (AAM) or encephalitis. Enterovirus detection was performed by isolation on cell culture (MRC5, BGM, Hep 2 e VERO) according to World Health Organization recommended protocols, and molecular methods based on reverse transcription (RT)-PCR. Isolates were identificated by indirect immunofluorescence staining with commercially available monoclonal antibodies and serotype identification was performed by seroneutralization (Lim and Benyesh-Melnick) of the cytopathic effect using pools of specific antisera.

Twenty-six patients (2.1\%), 15 males and 21 females, were found positive for Enterovirus with at least one of the test used.The average age was 23 (6-43).A total of 26 Non-Polio Enterovirus (NPEV) strains were isolated (II Echovirus, 5 Coxsackievirus, 10 not identified); the dominant strain of the outbreak was identified as a human Echovirus 6 (4 cases) followed by Echo 3I (3), Coxsackie B5 (3), Echo I7 (2) Echo 9, II, (I) and Coxsackie Al6 (I).

The yearly distribution of positive cases was homogeneous; a seasonal variation was noted with a predominance in summer-autumn; the higher transmission period starts in May and peaks in June-July; sporadic cases were also observed in winter and spring.

\section{INTRODUZIONE}

La Meningite Asettica Acuta (AAM) è una patologia ubiquitariamente diffusa fonte di $25000-50000$ ricoveri / anno nei soli Stati Uniti. (10) Circa 1'80\% dei casi di AAM sono causate da Enterovirus (14). Tra questi un ruolo preponderante è svolto dai Non Polio Enterovirus (NPEV): Coxsackievirus ed Echovirus (4), che sono causa anche di Encefaliti nonché di patologie di altri distretti corporei (Malattie respiratorie, malattie febbrili, esantematiche, mio-pericarditi e paralisi) (8).

NPEV si trasmettono per via orofecale e colpiscono soprattutto nei mesi estivi e autunnali interessando tutte le fasce di età; la popolazione pediatrica in particolare è la più frequentemente colpita. Le manifestazioni cliniche neurologiche associate all'infezione sono sì severe ma raramente fatali, in individui con sistema immunitario efficiente $(1,6)$. I sintomi durano per lo più 7-10 giorni dal momento dell'infezione (9).

Sebbene al momento non sia ancora disponibile una terapia antivirale specifica per le malattie da Enterovirus, la precoce individuazione di infezioni da essi provocate, può fornire informazioni utili alla gestione di un paziente o di una epidemia comunitaria con caratteristiche compatibili. Essa può inoltre servire ad evitare la somministrazione di una terapia antibiotica inutile e indesiderabile e a limitare l'entità dei test diagnostici effettuati in occasione di epidemie sospette.

Inoltre, è possibile sottoporre ad indagini e allertare persone ritenute essere a rischio di infezione (5). Non si può peraltro escludere che possa fra non molto rendersi disponibile una terapia antivirale specifica (12).

I ceppi di NPEV maggiormente isolati e tipizzati in casi di AAM sono Echovirus: il Sistema Nazionale di Sorveglianza per NPEV americano (NSSE), in uno studio multicentrico condotto tra gli anni 1999 e 2003 ha registrato una alta incidenza di Echovirus tipo $9(30 \%)$ e $30(36 \%)$, seguiti da Coxsackievirus B1 (8\%), Echovirus 7 (3\%), Coxsackievirus A9 (3\%), Enterovirus 71 (3\%), Coxsackievirus B4 (4\%) ed Echovirus 5 (2\%) (11). Uno studio analogo condotto nel bacino mediterraneo rivela dati pressoché sovrapponibili con alte percentuali di incidenza per Echovirus 6, 11 e 30 (2). Resta indiscusso anche 
nella vicina Francia il ruolo primario giocato dagli Echovirus quale causa di AAM (3).

Nel nostro studio retrospettivo abbiamo cercato di valutare l'incidenza dei casi di AAM e/o encefalite da NPEV da noi diagnosticati, in pazienti provenienti da aree differenti del territorio piemontese, in 5 anni di osservazione (2000-2004), al fine di confrontare i nostri dati con quelli della letteratura, anche in relazione alle metodiche di routine impiegate.

\section{MATERIALIE METODI}

Afferiscono al nostro centro campioni provenienti da 15 Ospedali Piemontesi e della Valle d'Aosta con un bacino di utenza complessivo di circa 4000000 di persone.

Abbiamo complessivamente esaminato 1232 Liquidi Cerebrospinali (CSF) provenienti da pazienti ricoverati nel periodo compreso tra gennaio 2000 e dicembre 2004 con diagnosi di AAM, encefalite o meningoencefalite di sospetta origine virale.

In 676 campioni gli Enterovirus sono stati ricercati unicamente mediante l'isolamento virale; in 173 mediante PCR, in 383 con entrambi i metodi. I protocolli operativi per gli isolamenti sono stati allestiti considerando le raccomandazioni e le linee guida per la diagnosi di Enterovirus dettate dal WHO (13).

I campioni per l'isolamento virale sono stati inoculati in linee cellulari MRC-5, BGM, Hep2 e Vero ed incubate a $37^{\circ} \mathrm{C}$ in termostato a $\mathrm{CO}_{2}$ $(5 \%)$; i tubi quotidianamente osservati al micro- scopio per la ricerca dell' effetto citopatico (CPE) per la durata di 21 giorni. In presenza di effetto citopatico l'identificazione di specie è stata eseguita mediante immunofluorescenza indiretta con anticorpi monoclonali anti-Enterovirus (ditta: DAKO Cytomation) e anti-Coxsackievirus, Echovirus e Poliovirus (Chemicon International Inc.). L'ulteriore tipizzazione di Coxsackievirus e Poliovirus era ancora eseguita mediante Immunofluorescenza indiretta (Chemicon International Inc,) mentre gli Echovirus venivano tipizzati mediante saggio di neutralizzazione (Lim and Benyesh-Melnick).

La ricerca del genoma virale è stata eseguita mediante RT-PCR-nested utilizzando il kit commerciale "Nested Enterovirus" fornito dalla ditta Amplimedical, previa estrazione con guanidina Idrocloruro e successiva precipitazione degli acidi nucleici con etanolo (kit commerciale Extragen-Amplimedical).

La procedura prevedeva l'esecuzione di due successive reazioni di amplificazione (nested) utilizzando coppie di primer differenti specifici per la regione genomica 5'UTR degli Enterovirus (7). La sensibilità della reazione è pari a circa 200 virus/ml e i primer scelti permettono l'amplificazione di tutti i sierotipi virali.

La rivelazione finale veniva effettuata mediante separazione elettroforetica in gel di agarosio $2 \%$. I ceppi identificati non erano ulteriormente tipizzati.

L'analisi statistica veniva effettuata con il test del chi quadro.

Tabella I. Ripartizione dei prelievi e dei casi positivi per NPEV dal 2000 al 2004 nel Laboratorio di virologia dell'Ospedale Amedeo di Savoia di Torino

\begin{tabular}{|c|c|c|c|c|c|c|c|c|c|c|c|c|}
\hline Anno & & 2000 & & 2001 & & 2002 & & 2003 & & 2004 & 200 & $0-2004$ \\
\hline $\begin{array}{l}\text { materiale } \\
\text { CSF }\end{array}$ & $\begin{array}{l}N \\
235\end{array}$ & \begin{tabular}{|c|} 
NPEV \\
Positivi \\
2 \\
$(0.8 \%)$
\end{tabular} & $\begin{array}{l}N \\
267\end{array}$ & $\begin{array}{c}\text { NPEV } \\
\text { Positivi } \\
\mathbf{3} \\
(1.1 \%)\end{array}$ & $\begin{array}{l}N \\
236\end{array}$ & \begin{tabular}{|c} 
NPEV \\
Positivi \\
I2 \\
$(5.1 \%)$
\end{tabular} & $\begin{array}{l}N \\
291\end{array}$ & \begin{tabular}{|c} 
NPEV \\
Positivi \\
4 \\
$(1.3 \%)$
\end{tabular} & $\begin{array}{l}N \\
203\end{array}$ & $\begin{array}{l}\text { NPEV } \\
\text { Positivi } \\
5 \\
(2.4 \%)\end{array}$ & $\begin{array}{l}N \\
I 232\end{array}$ & $\begin{array}{c}\text { NPEV } \\
\text { Positivi } \\
26 \\
(2.1 \%)\end{array}$ \\
\hline
\end{tabular}

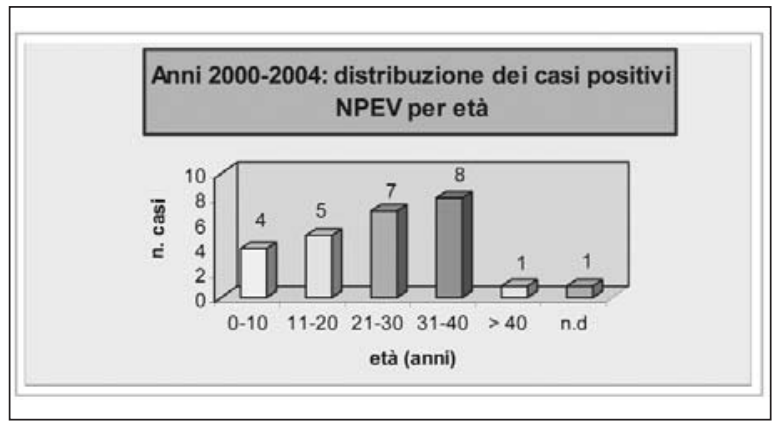

Figura I. Distribuzione per età dei casi positivi da NPEV dal 2000 al 2004 nel Laboratorio di virologia dell'Ospedale Amedeo di Savoia di Torino

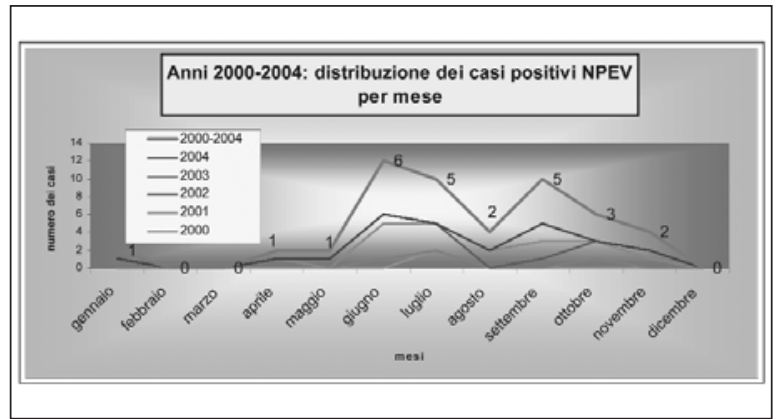

Figura II. Distribuzione mensile dei casi di NPEV isolati da liquor dal 2000 al 2004 nel Laboratorio di virologia dell'Ospedale Amedeo di Savoia di Torino 


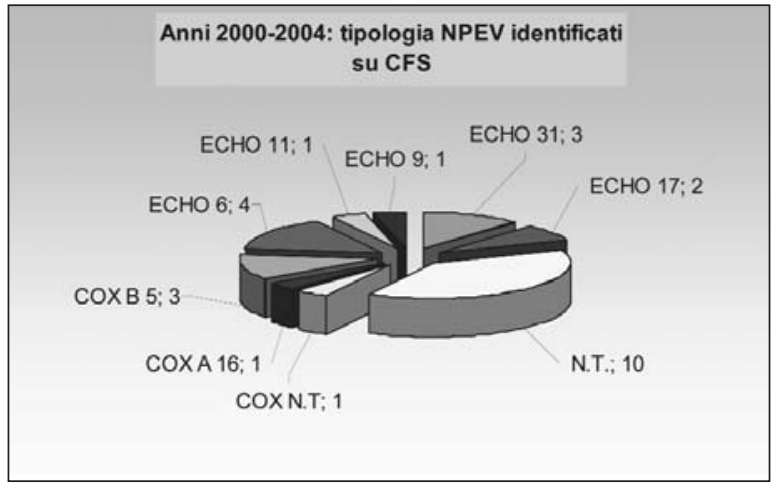

Figura III. Tipizzazione dei ceppi di NPEV isolati da liquor dal 2000 al 2004 nel Laboratorio di virologia dell'Ospedale Amedeo di Savoia di Torino

\section{RISULTATI}

Abbiamo complessivamente dimostrato 26 casi di Infezione del Sistema Nervoso Centrale da NPEV che rappresentavano il $2.1 \%$ dell'intera casistica; la distribuzione annuale variava dallo $0.8 \%$ dell'anno 2000 al 5.1\% del 2002; le differenze rilevate negli anni non risultavano statisticamente significative (tabella 1).

Da una analisi effettuata in base al territorio di provenienza emergeva una incidenza pari a 1 caso ogni 100000 abitanti/anno nel territorio canavesano afferente al reparto di neurologia dell'Ospedale di Ivrea (Reparto con il maggior numero di casi positivi).

L'età dei pazienti (15 maschi ed 11 femmine) variava da 6 a 43 anni (media 23 anni) con una prevalenza nella quarta decade di vita (31-40 anni) (figura I).

Il periodo di insorgenza della patologia era concentrato in massima parte nei mesi di giugno, luglio e settembre con rispettivamente 6,5 e 5 casi.

I rimanenti casi erano distribuiti uniformemente negli altri periodi dell'anno ad eccezione dei mesi di dicembre, febbraio e marzo (figura II).

L'identificazione di specie, eseguita sui 16 ceppi isolati in coltura evidenziava una prevalenza di Echovirus (68.8\%) rispetto a Coxsackievirus $(31.2 \%)$.

Con l'ulteriore sierotipizzazione Echovirus tipo 6 risultava il più frequentemente isolato (n. 4 casi) seguito da Coxsackievirus B5 ed Echovirus 31 (n. 3 casi ), Echovirus 17 (n. 2 casi), Echovirus 9, Echovirus 11, e Coxsackievirus tipo A16 (n. 1 caso).

Un ceppo identificato come Coxsackievirus non è stato ulteriormente tipizzato.

Non è stato possibile tipizzare i 10 ceppi ritrovati soltanto in PCR (colture non eseguite o negative) (figura III).
Relativamente ai metodi di indagine, su 14 campioni positivi contemporaneamente indagati mediante PCR e colture virali, 5 sono risultati positivi con entrambe le metodiche, 7 con la sola PCR e 2 con le sole colture virali.

\section{DISCUSSIONE E CONCLUSIONI}

Le infezioni del SNC da NPEV si confermano una realtà endemica nel territorio piemontese; il tasso di isolamento da noi rilevato è risultato tuttavia assai variabile a seconda degli ospedali da noi considerati, ciò potrebbe essere messo in relazione alle sensibilità diagnostiche (scelta dei pazienti, tempi, tipologia e modalità di raccolta e trasporto dei campioni) di ciascun centro, piuttosto che ad una reale variazione di incidenza tra le varie aree piemontesi.

Riteniamo che l'incidenza di 1 caso/100000 abitanti/anno da noi ritrovata possa comunque essere sottostimata, e che il ricorso a tutti gli strumenti diagnostici disponibili (isolamento + ricerca del genoma virale da liquor, essudato faringeo, e feci ed eventuale ricerca degli anticorpi specifici) potrebbe far salire tale stima.

La stagionalità estivo-autunnale coincide con i dati di letteratura, poiché tuttavia casi sporadici possono comparire anche nei mesi invernali e primaverili, riteniamo utile perseguire tale diagnostica lungo tutto il corso dell'anno.

Relativamente all'età, la relativa povertà di casi pediatrici è probabilmente da attribuirsi allo scarso numero di campioni da noi analizzati per questa fascia d'età.

Echovirus 6, 11, e Coxsackie B5 si confermano tra i virus maggiormente isolati, mentre non abbiamo ritrovato alcun Echovirus 30 (segnalato in molte casistiche come il più frequente); ciò potrebbe ascriversi ai lunghi intervalli che intercorrono normalmente (7-9 anni) tra una epidemia e l'altra (4). La nostra casistica, distribuita su 5 anni, risulta significativa; varrebbe la spesa poter allargare il raggio di osservazione confrontandola con dati provenienti da altre regioni italiane, magari con differenti condizioni climatiche, al fine di ottenere un panorama quanto più possibile completo del territorio nazionale.

\section{BIBLIOGRAFIA}

1. Abzung MJ. Presentation, diagnosis, and management of enterovirus infection in neonates. Pediatr. Drugs. 2004; 6(1): 1-10.

2. Bahri O, Rezig D, Nejma-Oueslati BB, et al. Enteroviruses in Tunisia: virological surveillance over 12 years (1992-2003). J Med Microbiol 2005 Jan; 54 (Pt 1): 63-9.

3. Bernit E, de Lamballerie X, Zandotti C, et al. 
Prospective investigation of a large outbreak of meningitis due to echovirus 30 during summer 2000 in Marseilles, France. Med 2004Jul; 83(4): 245-53.

4. Cermelli C, Portolani M. Eziologia e diagnostica delle infezioni del sistema nervoso centrale: agenti virali. Microbiologia Medica 1999; 12 (2); 87-92.

5. Cherry JD. Non polio Enteroviruses: Coxsackievirus, Echoviruses and Enteroviruses. In: Feigin RD, Cherry JD, eds, Textbook of pediatric infectious diseases. Philadelphia: WB Saunders, 1981: 1316-65.

6. Cree BC, Bernardini GL, Hays AP, Lowe G. A fatal case of Coxsackievirus B4 meningoencephalitis. Arch Neurol 2003 Jan; 60(1): 107-12.

7. Kuan MM. Detection and rapid differentation of human Enteroviruses following genomic Amplification .J Clin Microbiol 1997; 35: 2598-601.

8. Melnick JL. Enteroviruses: Polioviruses, Coxsackievirus, Echoviruses, and newer Enteroviruses. In: Fields B.N. et al., eds. Fields Virology. Philadelphia: Lippincott-Raven, 1996; 655-712.

9. Morens DM, Pallansch MA. Human enterovirus infections. Epidemiol 1995; 3-23.

10. Oberste MS, Maher K, Kennett ML, et al. Molecular epidemiology and genetic diversity of Echovirus type 30 (E30): genotypes correlate with temporal dynamics of E30 isolation. J Clin Microbiol 1999; 37: 3928-33.

11. Outbreaks of Aseptic Meningitis Associated with echoviruses 9 and 30 and Preliminary Surveillance Reports on Enterovirus Activity. CDC, United States, August 2003; 52(32); 761-4.

12. Rotbart HA, Webster AD. Treatment of potentially life-threatening enterovirus infections with pleconaril. Clin Infect Dis 2001; 32: 228-35.

13. www.wpro.who.int/NR/rdonlyres/CB0962C1-8612-41E0-B81F29E51882BF64/0/MTGRPT_Polio_CellCulture2003.pdf

\section{Pietro Giorgio Pistono}

Via Ivrea n. 23

10010 Fiorano Canavese (TO)

Tel. 0114393962 (838); Fax 0114393820

E-mail: pistono@asl3.to.it 